\title{
Optical Coherence Elastography for Non-Invasive Monitoring of Tumor Elasticity under Chemotherapy: Pilot Study
}

\author{
DOI: $10.17691 / \mathrm{stm} 2018.10 .3 .5$
}

Received May 16, 2018

A.A. Plekhanov, PhD Student, Institute of Biomedical Technologies ${ }^{1}$;

E.V. Gubarkova, PhD, Researcher, Scientific Laboratory of Optical Coherence Tomography, Institute of Biomedical Technologies ${ }^{1}$;

A.A. Sovietsky, PhD Student²; Senior Research Assistant, Laboratory of Wave Methods for Studying Structurally Inhomogeneous Media;

V.Y. Zaitsev, DSc, Head of the Laboratory of Wave Methods for Studying Structurally Inhomogeneous Media2;

L.A. Matveev, PhD, Senior Researcher, Laboratory of Wave Methods for Studying Structurally Inhomogeneous Media';

A.L. Matveyev, PhD, Senior Researcher, Laboratory of Multidimensional Signal Processing2;

L.B. Timofeeva, PhD, Assistant, Department of Histology with Cytology and Embryology';

S.S. Kuznetsov, MD, DSc, Head of the Department of Pathological Anatomy';

E.V. Zagaynova, MD, DSc, Professor of Russian Academy of Sciences, Director of the Institute

of Biomedical Technologies ${ }^{1}$;

N.D. Gladkova, MD, DSc, Professor, Head of the Scientific Laboratory of Optical Coherence Tomography, Institute of Biomedical Technologies";

M.A. Sirotkina, PhD, Senior Researcher, Scientific Laboratory of Optical Coherence Tomography ${ }^{1}$

${ }^{1}$ Privolzhsky Research Medical University, 10/1 Minin and Pozharsky Square, Nizhny Novgorod, 603005, Russia;

${ }^{2}$ Federal Research Center Institute of Applied Physics of the Russian Academy of Sciences, 46 Ul'yanova St.,

Nizhny Novgorod, 603950, Russia

The aim of the study was to evaluate a novel non-invasive method — optical coherence elastography (OCE) - for detecting early changes in the elasticity of tumor tissue in response to chemotherapy.

Materials and Methods. Female BalB/C mice were used in this experimentation. Cultured breast cancer cells (the 4T1 line) were implanted onto the surface of the mouse ear. The experimental animals were randomly separated into two groups: the control group ( $n=5$ ) and the therapeutic group $(n=5)$; the latter one received chemotherapy with cisplatin injected intraperitoneally at a dose of $6 \mathrm{mg} / \mathrm{kg}$. We then studied the elastic properties of the tumor tissue using a spectral multimodal optical coherence tomograph (Institute of Applied Physics of the Russian Academy of Sciences, Russia) that allowed for measuring the mechanical characteristics in terms of elastography. The compression mode of the OCE is based on estimating the inter-frame variation gradient of the OCE signal phases when tissue images are pairwise compared in the process of tissue deformation. By using a silicone layer for calibration we were able to determine the absolute values of the tissue stiffness (the Young's modulus of elasticity). The stiffness distribution across the tissue sample is displayed by images with a pseudocolor palette.

Results. The efficacy of chemotherapy with cisplatin was evaluated by the standard technique (kinetics of tumor growth), and then verified by histological analysis. Throughout the study, the tumor growth rate in the control group was significantly $(p<0.05)$ higher than that in the therapeutic group. By means of OCE significant differences $(p<0.05)$ in the stiffness of the tumor tissue were found between the therapeutic and control groups of animals already on day 5 after the start of chemotherapy.

By the end of the treatment, the OCE showed the lowest values of tumor stiffness, which correlated with the existence of extensive necrosis as confirmed by histology.

Conclusion. During chemotherapy, OCE can be used for in vivo monitoring of tumor stiffness as an index of treatment efficacy.

Key words: experimental tumor model; breast cancer; 4T1 mouse tumor model; optical coherence tomography; OCT; optical coherence elastography; OCE; cisplatin.

\section{Introduction}

Breast cancer remains the most common type of cancer in women. In $10 \%$ of cases, a triple negative form of breast cancer is diagnosed; there, the tumor cells lack the HER2 membrane protein, estrogen, and progesterone receptors, which make the targeted therapy useless. In case of triple-negative breast cancer, chemotherapy is of little efficacy too [1].

In experimental oncology, the efficacy of antitumor

Corresponding author: Anton A. Plekhanov, e-mail: strike_gor@mail.ru 
drugs is tested in animal tumor models [2]. The mouse tumor model 4T1 closely approximates the characteristics of human breast cancer. The 4T1 tumor is highly tumorigenic and invasive and, unlike most tumor models, can spontaneously metastasize from the primary tumor [3].

Cisplatin has been shown to effectively suppress 4T1 cancer [4]. Cisplatin, the chloride-ammonia complex of divalent platinum, is a cytostatic antitumor drug. The drug bifunctionally alkylates DNA strands, inhibits the biosynthesis of nucleic acids and thereby causes regression of tumor cells. The tumor cell death under chemotherapy occurs mainly by necrosis $[5,6]$. Necrotic tumor cells disintegrate and thus change the entire structure of the tumor tissue that leads to biomechanical properties changes, which can be measured to serve a marker of tumor degradation.

In recent years, biomechanical properties of breast tumors have been studied by ultrasonic elastography. The method provides for a qualitative and/or quantitative analysis of tissue elasticity based on the measurement of the Young's modulus. This approach has found its use for monitoring and predicting the results of neoadjuvant chemotherapy [7]. However, the relatively low spatial resolution of the current ultrasound-based elastography [8] restricts its diagnostic value.

Recently, a number of reports demonstrated the benefits of using optical coherence elastography (OCE) with a micron-level spatial resolution for intraoperative detection of surgical margins in breast cancer [9], for the differential diagnosis of benign and malignant neoplasms in the human breast tissue [10, 11], as well as for evaluation of chemotherapy efficacy in tumor models [12, 13].

In the present study, OCE elastography was used for live detection of an early response of the 4T1 murine tumor to chemotherapy.

The aim of the study was to evaluate a novel noninvasive method - optical coherence elastography for detecting early elasticity changes in tumor tissue in response to chemotherapy.

\section{Materials and Methods}

Animal tumor models. Female BalB/C mice were supplied by the lab animal breeding farm in Pushchino (Moscow region, Russia). The order No.199H "On the Approval of the Rules of Good Laboratory Practice" [14] and the International Guiding Principles for Biomedical Research Involving Animals (CIOMS and ICLAS, 2012) [15], as well as the ethical principles established by the European Convention for the Protection of Vertebrate Animals used for Experimental and other Scientific Purposes (Strasbourg, 2006), were strictly observed. The permission from the Ethical Committee of the Privolzhsky Research Medical University to conduct these specific experiments was obtained.

4T1 cells culture were implanted onto the surface of the mouse ear at a concentration of $2 \cdot 10^{5}$ cells $/ 20 \mu \mathrm{l}$ of phosphate buffer solution [16]. Morphological structure and other details of this tumor model are described in [3]. As shown earlier [17, 18], the $4 \mathrm{~T} 1$ model is a triple negative tumor, morphologically similar to the duct breast cancer and characterized by a high degree of malignancy and high metastatic activity.

It is important for the present OCE study that this tumor is located on the ear surface and allows for accurately measuring its size, visually assessing its growth and monitoring the changes in the tissue elasticity [19]. All the experimentation was performed under general anesthesia $(0.05 \mathrm{ml}$ of Zoletil $100+$ Rometar $2 \%$ in a $1: 1$ ratio).

Chemotherapy. The experimental animals were randomly divided into two groups: the control group $(n=5)$ and the therapeutic group $(n=5)$; the latter one received the cytostatic chemotherapeutic drug cisplatin at a dose of $6 \mathrm{mg} / \mathrm{kg}$ intraperitoneally, according to the earlier proposed treatment regimen [20]. Cisplatin was administered on day 2 post tumor inoculation. The subsequent therapy was carried out on days $3,5,7,10$, and 12, in total, 6 treatment sessions.

Criteria of the antitumor efficacy. During the first 14 days, the therapeutic effect by following tumor growth kinetics in the control and therapeutic groups was assessed. The tumor volume was determined using the formula: $\mathrm{V}_{t u}=\mathrm{p} / 6 \mathrm{a} \cdot b \cdot c$, where $a, b, c$ - length, width and depth of the tumor as measured with a caliper. Since at the baseline (day 0 ), the tumor volume varied greatly from mouse to mouse, the values of tumor volume on days 1-14 per the baseline value to get the ratio $V / V_{0}$ for the very same mouse were normalized.

Histological examination. To assess the degree and the mechanism of the tumor damage under chemotherapy, the areas of necrosis in the therapeutic and experimental groups were compared. To that end of the study, on day 14 after the tumor implantation the animals were euthanized by dislocating the cervical vertebrae. The histological slides prepared from the tumor tissues were stained by hematoxylin and eosin. The Avtandilov's morphometric technique was used to measure the areas of necrosis and the percentage of tumor cells with nucleus and cytoplasm irreversible changes (karyolysis, karyopyknosis, karyorrhexis, vacuolization of the nucleus and the cytoplasm). The resulting cell counts were expressed per total area of the histological slide. At least three histological specimens from each tumor were examined.

Optical coherent elastography. The elasticity characteristics of tumor tissues were measured using a spectral multimodal optical coherence tomograph (Institute of Applied Physics of the Russian Academy of Sciences, Russia) in the elastography mode. The multimodal optical coherence tomograph is based on the spectral principle of signal reception and can provide 20,000 A-scans per second [21, 22]. In this study, we used a broadband near infrared signal $(1.3 \mu \mathrm{m})$ with 
a power of about $15 \mathrm{~mW}$. The device has a spatial resolution of $15 \mu \mathrm{m}$ wide and about $10 \mu \mathrm{m}$ (in air) deep [23].

The compression OCE mode is based on estimating the inter-frame variation gradient of the OCE signal phases when tissue images are pairwise compared in the process of tissue deformation. To process the information brought by the OCE signal, an improved (hybrid) version of the phase-associated monitoring of tissue scattering [24] and a robust vector method for estimating phase gradients $[25,26]$ were used. A calibration (silicone) layer with a known stiffness (200 kPa) was placed on the tissue surface to help calculate the absolute values of the tissue stiffness (the Young's modulus of elasticity) from the ratio of silicone deformation to tissue deformation. The resulting image is represented with a pseudocolor palette, where the rigid (poorly deformed) areas are shown in blue, and the soft (markedly deformed) areas are red. The principle behind obtaining the images has been described earlier $[25,26]$. The OCE-related measurements were carried out on day $3,5,7,10,12$, and 14 after the tumor initiation. When an OCT probe was used to study the mechanical properties of the tumor tissue, the calibration silicone was placed on top of the tissue sample, and compression was applied to both materials. The primary data were processed in the MATLAB environment to result in stiffness distribution maps for the tumor tissue specimen [27].

\section{Results}

Changes in tumor volume after chemotherapy. In the tumor growth kinetics, statistically significant differences were found between the control and therapeutic groups $(p \leq 0.05)$. Throughout the study, the growth rate of tumors in the control group was significantly higher than that in the therapeutic group (Figure 1). Thus, three days after the first chemotherapy session, the average tumor volume in the therapeutic group increased 1.83-fold from day 0 , while the average tumor volume in the control group increased 4-fold.

On day 14, after six chemotherapy sessions, the following dynamics was observed: the average tumor volume in the therapeutic group increased 9.84-fold from day 0 as compared with a 40 -fold increase in the control group ( $p \leq 0.05)$. The significant difference in the tumor growth rate between the control and therapeutic groups indicated a chemotherapeutic effect of cisplatin.

Histological examination. In the histological preparations obtained on the final day of the experiment (day 14), the tumor tissue in the control group had a morphological structure with the characteristic predominance of parenchymal components (98-99\%) over stromal ones ( 1\%) (Figure $2(\mathrm{a})$ ). The stroma was represented by small diffuse bundles of collagen fibers occupying $1-2 \%$ of the neoplasm, and by tiny capillaries of the sinusoidal type. In the upper part of the slide, necrosis of epidermal cells and (partially) tumor cells was observed; the necrotic areas occupied up to $20 \%$ of the entire preparation (Figure 2 (c)).

In the therapeutic group, tumors were characterized by large foci of necrosis (up to $55 \%$ of the tumor area) associated with a loss of histological structure (Figure 2 (b), (d)); a moderate tissue swelling was also detected. Diapedesis, hemorrhages, stasis, sludge, and full-blooded capillaries were seen, mainly on the border between the tumor and normal ear tissue. In the remaining areas of the tumor tissue, about $10-25 \%$ of the cells showed irreversible changes in the nucleus and/or cytoplasm - e.g., karyolysis, karyopyknosis, karyorrhexis, and vacuolization (Figure 2 (e)). In about $25 \%$ of the cells, there were signs of an abnormal nucleus division such as non-viable forms of mitosis. The similar changes were detected both in the central parts of the tumors and in their periphery, in all animals of the therapeutic group.

Elastic properties of the tumor. For the first 4 days of the tumor growth, its volume was too small to perform accurate elastography. Therefore, reliable results
Figure 1. Kinetics of tumor growth under chemotherapy with cisplatin at a dose of $6 \mathrm{mg} / \mathrm{kg}$

The tumor volume $(\mathrm{V})$ at each time point was normalized to the initial value $\left(V_{0}\right)$ measured on day 0 ; the green arrows indicate the timing of cisplatin administration; * statistically significant difference from the control group $(p<0.05)$

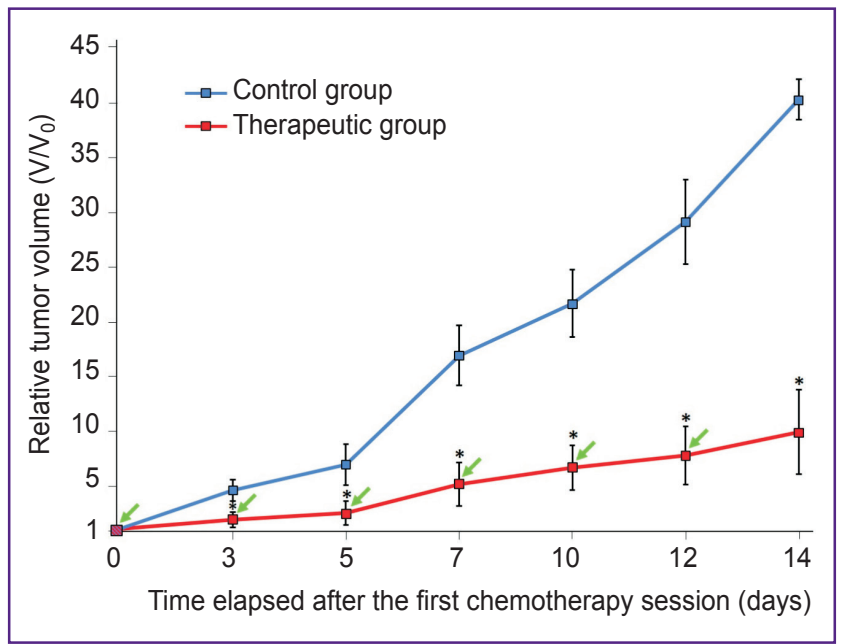

Optical Coherence Elastography for Non-Invasive Monitoring of Tumor Elasticity

CTM $\int 2018 \int$ vol. $10 \int$ No.3 


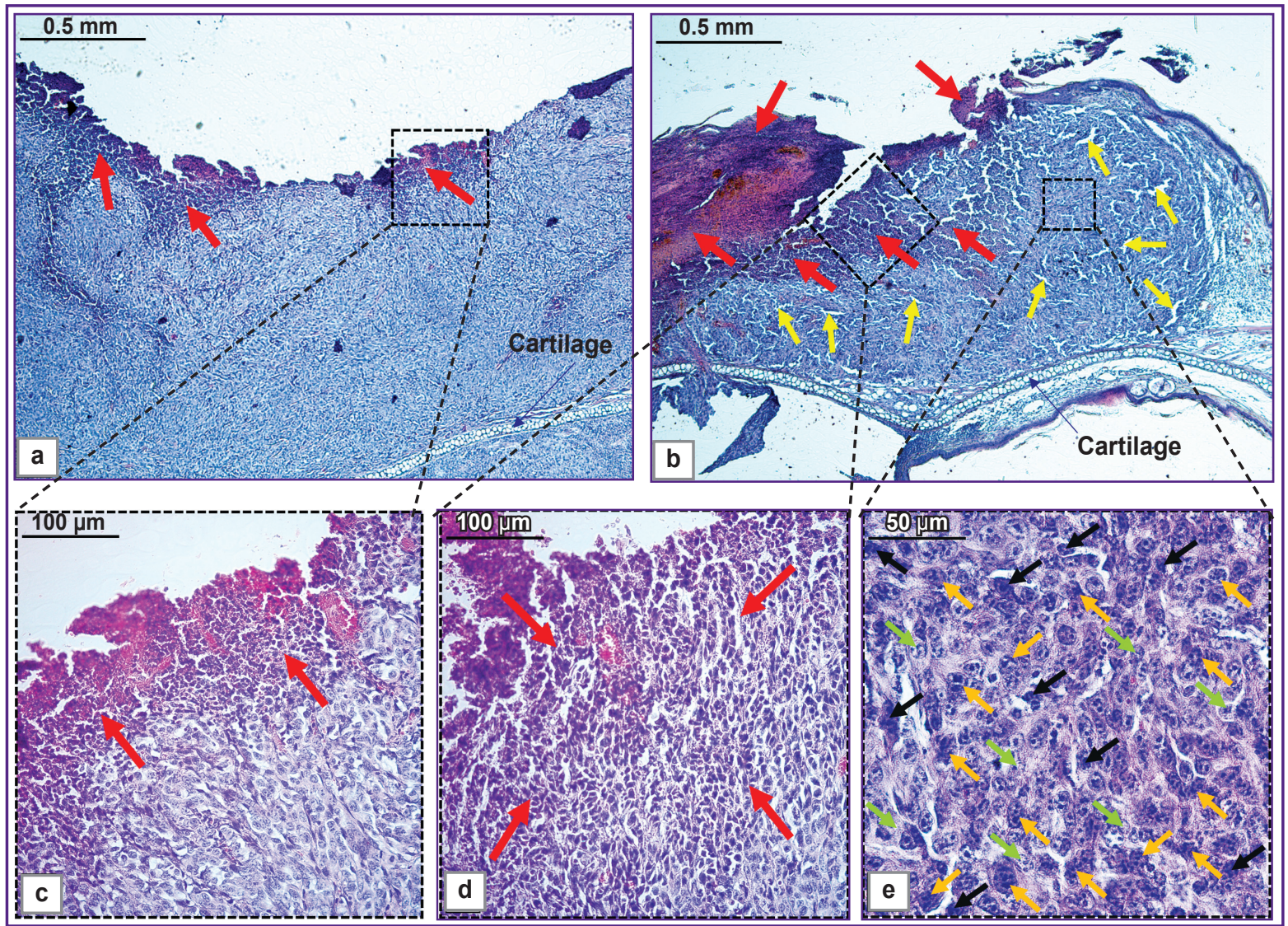

Figure 2. Histological examination of the 4T1 model:

(a) tumor from the control group; (b) tumor from the therapeutic group; (c) superficial necrosis in tumors from the control group; (d) total tumor necrosis in the therapeutic group; (e) karyopyknosis and karyolysis in tumors from the therapeutic group; the red arrows show areas of tumor necrosis, the yellow arrows indicate areas of edema in the tumor tissue, the orange arrows point to tumor cells with nuclear damage (karyorrhexis), the black arrows indicate karyopyknosis in tumor cells, the green arrows point to the areas of irreversible nuclear changes — karyolysis

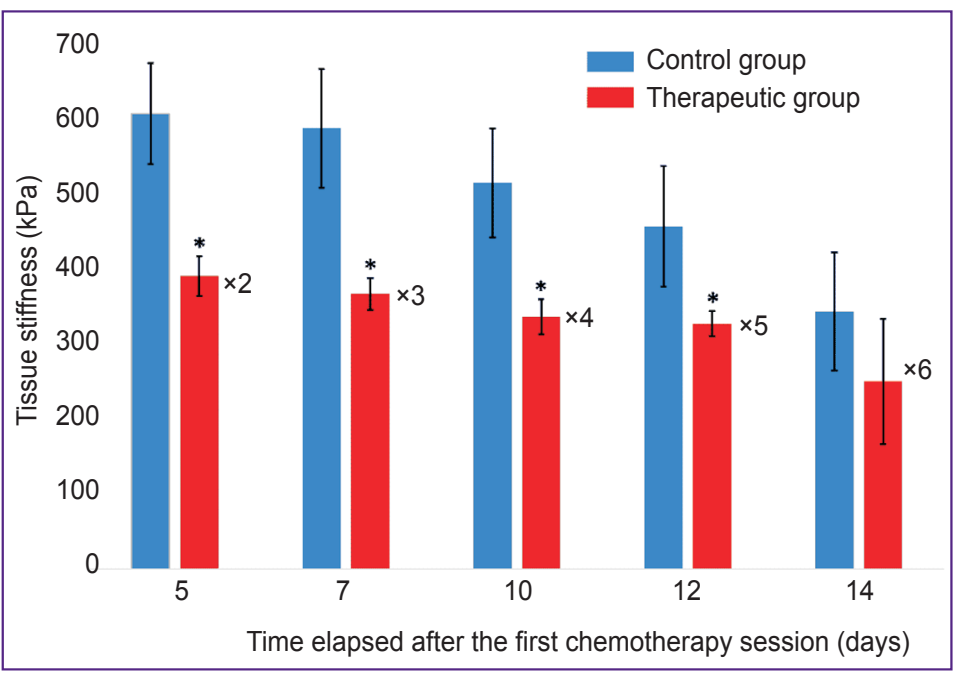

Figure 3. Change in the tumor stiffness during chemotherapy with cisplatin at a dose of $6 \mathrm{mg} / \mathrm{kg}$

$\times$ The number of chemotherapy sessions by a given time point;

* statistically significant difference from the control group $(p<0.05)$ were obtained only starting from day 5 of the experiment. In Figure 3, changes in the tumor stiffness without and with chemotherapy are shown. On day 5 , the tumors in the treatment group were significantly softer $(386 \pm 26 \mathrm{kPa})$ than those in the control group $(600 \pm 66 \mathrm{kPa})$ $(p \leq 0.05)$. This trend continued up to day 12 when the average stiffness of tumors in the therapeutic group was $323 \pm 16 \mathrm{kPa}$ versus $451 \pm 79 \mathrm{kPa}$ in the control group $(p \leq 0.05)$. By the end of the observation, the tumors in both groups had low stiffness values, which were most likely due to the developing necrosis of cancer cells, either spontaneous or chemotherapy-induced.

The elastographic maps showed that in control, the tumors had high stiffness values at the early growth period (Figure 4 (a)). Later on, softer areas appeared and stiffness decreased (Figure 4 (b), (c)). In the therapeutic group, low stiffness areas appeared at the early stages 


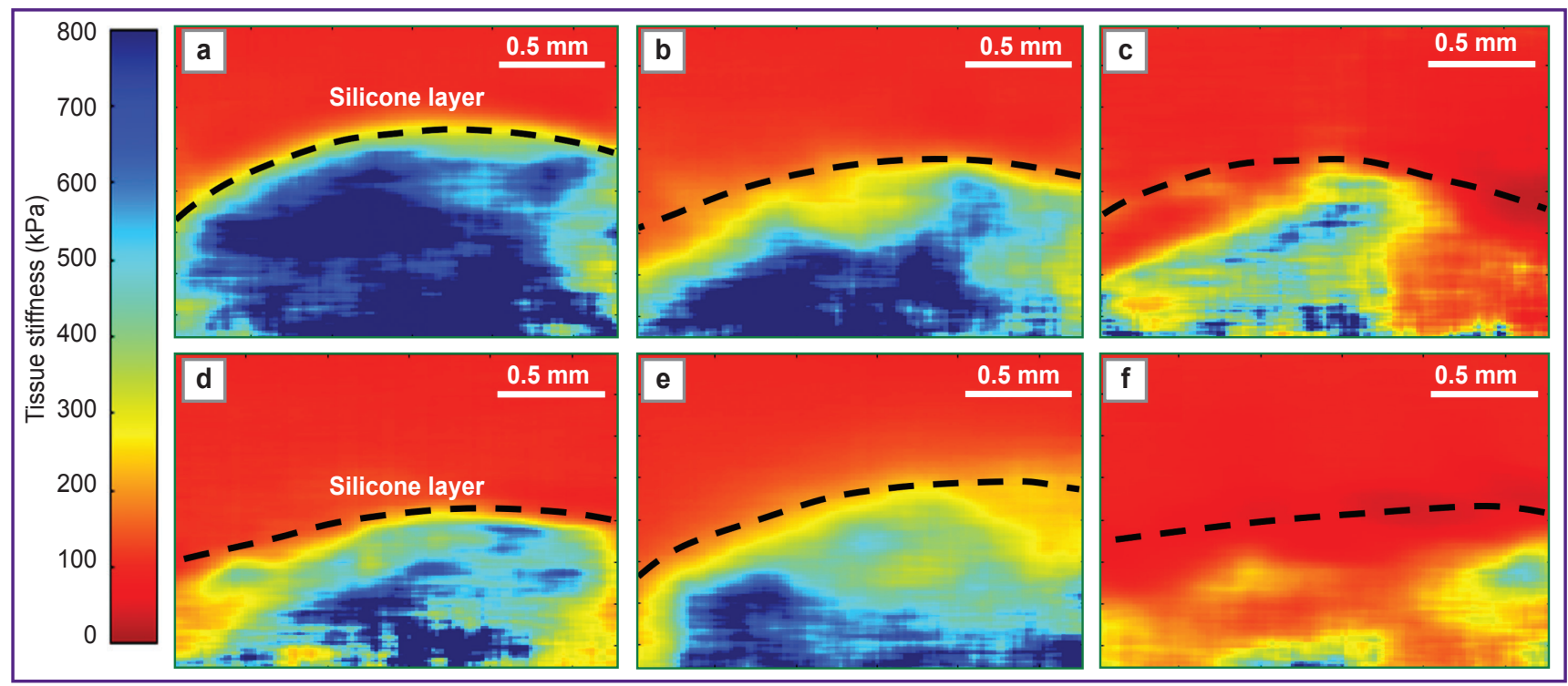

Figure 4. Elastographic maps of stiffness distribution in the tumor tissues of mice in the control and therapeutic groups: (a)-(c) changes in tumor stiffness in the control group as detected on days 5 (a), 10 (b), and 14 (c); (d)-(f) changes in tumor stiffness in the therapeutic group as detected on days 5 (d), $10(\mathrm{e})$, and 14 (f); the dotted line indicates the boundary between the calibration (silicone) layer and the tumor tissue; the boundary was determined by the structural images; on the left, the stiffness scale expressed in the units of the Young's modulus of elasticity $(\mathrm{kPa})$; the pseudocolor palette: blue color - the areas of the highest stiffness (about $800 \mathrm{kPa}$ ), yellow-green color - the medium stiffness (300-400 kPa) and red color - the softest (lowest stiffness) areas (about $0 \mathrm{kPa})$

of tumor growth (Figure 4 (d)), and thereafter, even lower stiffness values were detected (Figure 4 (e), (f)). After 14 days, a dense layer of dead epidermal cells developed on the tumor surface, which was typical for this murine tumor model. Because of that no further OCE measurements were feasible and the elastography studies were stopped.

\section{Discussion}

This is a preliminary research into the ability of the new method - OCE - to monitor antitumor therapy (in this case, with cisplatin) at an early stage.

To date, a number of non-invasive methods have been used to evaluate the efficacy of chemotherapeutic agents: among them, kinetics of tumor growth inhibition, changes in body weight, the life span and overall survival of experimental animals $[28,29]$. Yet, the gold standard has been and still is the morphological analysis of both the primary tumor and distant metastases in various organs [30]. The histological method though is logistically complicated and time-consuming because it requires the animals to be sacrificed at each timepoint, which considerably increases the total number of animals in the experiment. Therefore, the development of high-precision non-invasive techniques to follow the tumor growth during antitumor therapy is important.

In the present study, the standard methods of evaluating the antitumor activity confirmed that cisplatin exerted a noticeable chemotherapeutic effect on the 4T1 tumor. This was evidenced by the significantly lower rate of tumor growth in the therapeutic group. The results (see Figure 2) were in a good agreement with those reported by others $[4,20]$.

In the therapeutic group, the histological changes in the tumor tissues are similar to described in the literature [31]. Thus, Li et al. reported that after treatment by cisplatin in combination with doxorubicin, extensive areas of necrosis were observed in tumor cells; in other cells, various nuclear abnormalities - nuclear clamping and fragmentation - were revealed. Fragmentation of nuclei (karyorrhexis) may indicate the mechanism of apoptosis in tumor cells [32]. Other authors [32, 33] also demonstrated an apoptotic response in 4T1 tumor cells exposed to cisplatin in vitro. In our study, karyorrhexis was observed in tumors of the therapeutic group, which might indicate the mechanism of apoptotic cell death caused by cisplatin.

Although both necrosis and apoptosis can occur under exposure to chemotherapeutic drugs, the role of necrosis in tumor cell death is considered the most significant [6]. In line with this concept, the antitumor activity of chemotherapeutic agents is classified by the volume of tumor necrosis [34]: more than $90 \%$ of necrosis in a tumor is considered a good antitumor response. In our study on the 4T1 model, the histological tests revealed a moderate (55\% necrosis) antitumor effect of cisplatin.

The OCE measurements during the tumor growth allowed us to monitor its response to chemotherapy 
by changes in the mechanical stiffness of the tumor. Significant differences in the stiffness values were found between the therapeutic and control groups, and the stiffness value decreased during the treatment. These results are consistent with experimental and clinical studies using ultrasonic elastography, where changes in tumor stiffness were reported at 2 and 4 weeks after the initiation of chemotherapy for breast cancer [12] and rectal cancer [13]. In our study, the stiffness values differed between the two groups starting as early as day 5 after the tumor implantation. In this early period, the tumor stiffness changed only in the therapeutic group ( $p<0.05)$, while in the control group it remained unchanged.

Thus, the OCE method can be used for in vivo monitoring of early changes in tumor stiffness as an efficacy index of chemotherapy. Detailed interpretation of the stiffness distribution maps by comparing them with the histological preparations at different time-points will allow us to rationalize the use of OCE for non-invasively evaluating the efficacy of antitumor drugs.

\section{Conclusion}

Optical coherent elastography is able to detect a tumor softening, starting from day 5 of cisplatin chemotherapy. By the end of the treatment, the tumor stiffness decrease to its lowest level, which correlates with extensive necrosis, as revealed histologically. This allows us to propose the OCE as a reliable and sensitive method for non-invasive in vivo monitoring of biomechanical changes in tumors under chemotherapy.

Financial support. The development of the tumor model and the conduct of experimental studies on animals was made possible with the financial support from the President of the Russian Federation (grant No.MK-905.2017.7 for young scientists); The quantitative analysis of tissue elasticity using OCE was carried out with the financial support of the Russian Foundation for Basic Research within the scientific project No.1832-00608_mol_a. The adjustment of the elastography processing algorithm to the purpose of the present study was supported by the Russian Foundation for Basic Research and Nizhny Novgorod Government (grant No.18-42-520018).

Conflict of interest. The authors have no conflict of interest.

\section{References}

1. Rak molochnoy zhelezy [Breast cancer]. Pod red. Franka G.A., Zavalishinskoy L.E., Pozharisskogo K.M. [Frank G.A., Zavalishinskaya L.E., Pozharisskiy K.M. (editors)]. Moscow; 2014; 171 p.

2. Treshchalina E.M., Zhukova O.S., Gerasimova G.K., Andronova N.V., Garin A.M. Metodicheskie ukazaniya po izucheniyu protivoopukholevoy aktivnosti farmakologicheskikh veshchestv. V kn.: Rukovodstvo po eksperimental'nomu (doklinicheskomu) izucheniyu novykh farmakologicheskikh veshchestv [Methodological guidelines to study the antitumor activity of pharmacological substances. In: Guideline for experimental (preclinical) studying of new pharmacological substances]. Pod red. Habrieva R.U. [Habriev R.U. (editor)]. Moscow: Meditsina; 2005; p. 637-651.

3. Garcia C.M.S., de Araújo M.R., Lopes M.T.P., Ferreira M.A.N.D., Cassali G.D. Morphological and immunophenotipical characterization of murine mammary carcinoma 4t1. Braz J Vet Pathol 2014; 7(3): 158-165.

4. Li M., Tang Z., Zhang Y., Lv S., Li Q., Chen X. Targeted delivery of cisplatin by LHRH-peptide conjugated dextran nanoparticles suppresses breast cancer growth and metastasis. Acta Biomater 2015; 18: 132-143, https://doi. org/10.1016/j.actbio.2015.02.022.

5. Joo M.W., Kang Y.K., Yoo C.Y., Cha S.H., Chung Y.G. Prognostic significance of chemotherapy-induced necrosis in osteosarcoma patients receiving pasteurized autografts. PLOS One 2017; 12(2): e0172155, https://doi.org/10.1371/journal. pone.0172155.

6. Zhang J., Lou X., Jin L., Zhou R., Liu S., Xu N., Liao D.J. Necrosis, and then stress induced necrosis-like cell death, but not apoptosis, should be the preferred cell death mode for chemotherapy: clearance of a few misconceptions. Oncoscience 2014; 1(6): 407-422, https://doi.org/10.18632/ oncoscience.61.

7. Evans A., Armstrong S., Whelehan P., Thomson K., Rauchhaus P., Purdie C., Jordan L., Jones L., Thompson A., Vinnicombe S. Can shear-wave elastography predict response to neoadjuvant chemotherapy in women with invasive breast cancer? Br J Cancer 2013; 109(11): 2798-2802, https://doi. org/10.1038/bjc.2013.660.

8. Itoh A., Ueno E., Tohno E., Kamma H., Takahashi H., Shiina T., Yamakawa M., Matsumura T. Breast disease: clinical application of US elastography for diagnosis. Radiology 2006; 239(2): 341-350, https://doi.org/10.1148/radiol.2391041676.

9. Chin L., Latham B., Saunders C.M., Sampson D.D., Kennedy B.F. Simplifying the assessment of human breast cancer by mapping a micro-scale heterogeneity index in optical coherence elastography. J Biophotonics 2017; 10(5): 690-700, https://doi.org/10.1002/jbio.201600092.

10. Kennedy B.F., McLaughlin R.A., Kennedy K.M., Chin L., Curatolo A., Tien A., Latham B., Saunders C.M., Sampson D.D. Optical coherence micro-elastography: mechanical-contrast imaging of tissue microstructure. Biomed Opt Express 2014; 5(7): 2113-2124, https://doi.org/10.1364/boe.5.002113.

11. Kennedy B.F., McLaughlin R.A., Kennedy K.M., Chin L., Wijesinghe P., Curatolo A., Tien A., Ronald M., Latham B., Saunders C.M., Sampson D.D. Investigation of optical coherence microelastography as a method to visualize cancers in human breast tissue. Cancer Res 2015; 75(16): 3236-3245, https://doi.org/10.1158/0008-5472.can-14-3694.

12. Falou O., Sadeghi-Naini A., Prematilake S., Sofroni E., Papanicolau N., Iradji S., Jahedmotlagh Z., Lemon-Wong S., Pignol J.P., Rakovitch E., Zubovits J., Spayne J., Dent R., Trudeau M., Boileau J.F., Wright F.C., Yaffe M.J., Czarnota G.J. Evaluation of neoadjuvant chemotherapy response in women with locally advanced breast cancer using ultrasound elastography. Trans/ Oncol 2013; 6(1): 17-24, https://doi. org/10.1593/tlo.12412.

13. Rafaelsen S.R., Vagn-Hansen C., Sørensen T., Lindebjerg J., Pløen J., Jakobsen A. Ultrasound elastography in patients with rectal cancer treated with chemoradiation. 
Eur J Radiol 2013; 82(6): 913-917, https://doi.org/10.1016/j. ejrad.2012.12.030.

14. Ministry of Health and Social Development of the Russian Federation. Order dated April 1, 2016, No. 199H "On the Approval of the Rules of Good Laboratory Practice".

15. International Guiding Principles for Biomedical Research Involving Animals. CIOMS and ICLAS; 2012.

16. Song H.-W., Lee S.-W., Jung M.-H., Kim K.R., Yang S., Won P.J., Jeong M.-S., Jung M.Y., Kim S. Optical monitoring of tumors in BALB/c nude mice using optical coherence tomography. Journal of the Optical Society of Korea 2013; 17(1): 91-96, https://doi.org/10.3807/josk.2013.17.1.091.

17. Pulaski B.A., Ostrand-Rosenberg S. Mouse 4T1 breast tumor model. Curr Protoc Immunol 2001; Chapter 20: Unit 20.2, https://doi.org/10.1002/0471142735.im2002s39.

18. Yang S., Zhang J.J., Huang X.-Y. Mouse models for tumor metastasis. Rational Drug Design. Methods in Molecular Biology (Methods and Protocols) 2012; 928: 221-228, https:// doi.org/10.1007/978-1-62703-008-3_17.

19. Sirotkina M.A., Matveev L.A., Shirmanova M.V., Zaitsev V.Y., Buyanova N.L., Elagin V.V., Gelikonov G.V., Kuznetsov S.S., Kiseleva E.B., Moiseev A.A., Gamayunov S.V., Zagaynova E.V., Feldchtein F.I., Vitkin A., Gladkova N.D. Photodynamic therapy monitoring with optical coherence angiography. Sci Rep 2017; 7: 41506, https://doi.org/10.1038/ srep41506.

20. Du J., Sun Y., Wang X.F., Lu Y.Y., Zhou Q.M., Su S.B. Establishment of an experimental breast cancer ZHENG model and curative effect evaluation of Zuo-Jin Wan. Evid Based Complement Alternat Med 2013; 2013: 324732, https://doi. org/10.1155/2013/324732.

21. Gelikonov V.M., Gelikonov G.V., Shilyagin P.A. Linearwavenumber spectrometer for high-speed spectral-domain optical coherence tomography. Optics and Spectroscopy 2009; 106(3): 459-465, https://doi.org/10.1134/s0030400x09030242.

22. Gelikonov V.M., Gelikonov G.V., Kasatkina I.V., Terpelov D.A., Shilyagin P.A. Coherent noise compensation in spectral-domain optical coherence tomography. Optics and Spectroscopy 2009; 106(6): 895-900, https://doi.org/10.1134/ s0030400x09060174.

23. Sirotkina M.A., Buyanova N.L., Kalganova T.I., Karabut M.M., Elagin V.V., Kuznetsov S.S., Snopova L.B., Gelikonov G.V., Zaitsev V.Yu., Matveev L.A., Zagaynova E.V., Vitkin A., Gladkova N.D. The development of the methodology of monitoring experimental tumors using multimodal optical coherence tomography: the choice of an optimal tumor model. Sovremennye tehnologii v medicine 2015; 7(2): 6-15, https:// doi.org/10.17691/stm2015.7.2.01.

24. Zaitsev V.Y., Matveyev A.L., Matveev L.A., Gelikonov G.V., Gubarkova E.V., Gladkova N.D., Vitkin A. Hybrid method of strain estimation in optical coherence elastography using combined sub-wavelength phase measurements and supra-pixel displacement tracking. J Biophotonics 2016; 9(5): 499-509, https://doi.org/10.1002/ jbio.201500203.

25. Zaitsev V.Y., Matveyev A.L., Matveev L.A.,
Gelikonov G.V., Sovetsky A.A., Vitkin A. Optimized phase gradient measurements and phase-amplitude interplay in optical coherence elastography. J Biomed Opt 2016; 21(11): 116005, https://doi.org/10.1117/1.jbo.21.11.116005.

26. Matveyev A.L., Matveev L.A., Sovetsky A.A., Gelikonov G.V., Moiseev A.A., Zaitsev V.Y. Vector method for strain estimation in phase-sensitive optical coherence elastography. Laser Physics Letters 2018; 15(6): 065603, https://doi.org/10.1088/1612-202x/aab5e9.

27. Zaitsev V.Y., Matveyev A.L., Matveev L.A., Gubarkova E.V., Sovetsky A.A., Sirotkina M.A., Gelikonov G.V., Zagaynova E.V., Gladkova N.D., Vitkin A. Practical obstacles and their mitigation strategies in compressional optical coherence elastography of biological tissues. J Innov Opt Health Sci 2017; 10(6): 1742006, https://doi.org/10.1142/ s1793545817420068.

28. Pandey A., Sarangi S., Chien K., Sengupta P., Papa A.L., Basu S., Sengupta S. Anti-platelet agents augment cisplatin nanoparticle cytotoxicity by enhancing tumor vasculature permeability and drug delivery. Nanotechnology 2014; 25(44): 445101, https://doi.org/10.1088/0957-4484/25/44/445101.

29. Li Q., Tian Y., Li D., Sun J., Shi D., Fang L., Gao Y., Liu $H$. The effect of lipocisplatin on cisplatin efficacy and nephrotoxicity in malignant breast cancer treatment. Biomaterials 2014; 35(24): 6462-6472, https://doi. org/10.1016/j.biomaterials.2014.04.023.

30. Yu H., Guo C., Feng B., Liu J., Chen X., Wang D., Teng L., Li Y., Yin Q., Zhang Z., Li Y. Triple-layered pHresponsive micelleplexes loaded with siRNA and cisplatin prodrug for NF-kappa B targeted treatment of metastatic breast cancer. Theranostics 2016; 6(1): 14-27, https://doi. org/10.7150/thno.13515.

31. Li M., Tang Z., Zhang D., Sun H., Liu H., Zhang Y., Zhang $Y$., Chen $X$. Doxorubicin-loaded polysaccharide nanoparticles suppress the growth of murine colorectal carcinoma and inhibit the metastasis of murine mammary carcinoma in rodent models. Biomaterials 2015; 51: 161-172, https://doi.org/10.1016/j.biomaterials.2015.02.002.

32. Sri H., Ratna A.S., Zalinar U., Edy M., Riris I.J. Brazilein in combination with cisplatin inhibit proliferation and migration on highly metastatic cancer cells, 4T1. Indonesian Journal of Biotechnology 2016; 21(1): 38-47, https://doi.org/10.22146/ ijbiotech.26106.

33. Izgi K., Iskender B., Sakalar C., Arslanhan A., Yuksek E.H., Hizar E., Canatan H. Effects of epirubicin and cisplatin against $4 \mathrm{~T} 1$ breast cancer cells are enhanced by Myrtucommulone-A. Anticancer Agents Med Chem 2017. 17(3): 404-414, https://doi.org/10.2174/187152061666616040 4110543.

34. Rosen G., Caparros B., Huvos A.G., Kosloff C., Nirenberg A., Cacavio A., Marcove R.C., Lane J.M., Mehta B., Urban C. Preoperative chemotherapy for osteogenic sarcoma: selection of postoperative adjuvant chemotherapy based on the response of the primary tumor to preoperative chemotherapy. Cancer 1982; 49(6): 1221-1230, https://doi,org/10 1002/10970142(19820315) 49:6<1221::aid-cncr2820490625>3.0.co;2-e. 